ISSN: 2637-9627

\title{
Outcomes for Newborns with Mild Hypoxic-Ischemic Encephalopathy: A Retrospective Study
}

\author{
Amani Saeed; Husam Salama*; Amr Moussa; Mai Al Qubasi; Safaa Al Saige; Shihab kannappillil; Sara Ahmed \\ Women's Wellness and Research Center (WWRC), Qatar.
}

\section{*Corresponding Author(s): Husam Salama}

Senior Neonatologist, Women wellness and research center-NICU, Hamad Medical Corporation. Doha, Qatar. Tel: 097455262159; Email: hsalama1@hamad.qa

Received: Aug 19, 2021

Accepted: Sep 08, 2021

Published Online: Sep 10, 2021

Journal: Annals of Pediatrics

Publisher: MedDocs Publishers LLC

Online edition: http://meddocsonline.org/

Copyright: (C) Salama H (2021). This Article is distributed under the terms of Creative Commons Attribution 4.0 International License

Keywords: Asphyxia neonatorum; Mild hypoxic ischemic encephalopathy; Therapeutic hypothermia; Newborn; Cooling.

Abbreviations: mHIE: mild Hypoxic Ischemic Encephalopathy; aEEG: amplitude Electroencephalogram; ABND: Abnormal Neuro-Developmental; MRI: Magnetic Resonance Imaging; NICU: Neonatal Intensive Care Unite; NND: Normal Neuro-Developmental; NIPPV: Noninvasive Positive Pressure Ventilation; NDI: Neuro-Developmental Impairment; TH: Therapeutic Hypothermia; WWRC: Women's Wellness Research Center.

\section{Abstract}

Background: HIE remains a significant cause of mortality and long-term disability in late preterm and term newborns. At birth, the only available distinction between mild, moderate, and severe HIE is based on the clinical ground. Nevertheless, mild HIE can be presented with subtle or subjective clinical features which may mislead the treating physician and delay his decision to intervene.

Methods: This is a retrospective descriptive study examined all inborn newborns $\geq 35$ weeks gestational age born at a single, tertiary level Neonatal Intensive Care Unit (NICU) in women's hospital. The study revised newborns who were admitted to NICU during the period from November 2014 till November 2020 under the diagnoses of mild HIE. The decision to start therapeutic hypothermia in cases of $\mathrm{mHIE}$ was off-label and it was taken according to the clinical judgment of the treating team.

Results: Out of the 265 newborns admitted with a history suggestive of HIE or neurological deficits, only 116 newborns matched the diagnosis of $\mathrm{mHIE}$ according to the above-mentioned exclusions. 19 newborns out of the 116 mHIE cases received therapeutic hypothermia. Antepartum and or intrapartum complications were recorded in 48 mothers including an infant of insulin-dependent diabetic mother 12 , pre-eclampsia 3 , cord prolapse 2 , shoulder dystocia 2, antepartum hemorrhage 8 , chorioamnionitis 6, poor CTG tracing 13, and ruptured uterus 2 .

Mean gestation was $38 \pm 2$ weeks, mean birth weight was $3.0 \pm 0.5 \mathrm{~kg}$, Cesarean section was $57 \%$ in the un-cooled group vs $75 \%$ in the cooled group. Mean Apgar score at 10 th minute was $7.9 \pm 1.8$ vs $5.3 \pm 2.2$ in the un-cooled vs cooled group, the $\mathrm{p}$-value is 0.002 . Arterial cord $\mathrm{pH}$ was $7.15 \pm 0.3$ vs $6.92 \pm 0.26$. The base deficit in the first-hour blood gas was $-7.83 \pm 5$ vs $-12 \pm 5.6(P=0.005)$. The Total number of cooled newborns was 19 (16\%). Respiratory support was required

Cite this article: Saeed A, Salama H, Moussa A, Qubasi MA, Saige SA, et al. Outcomes for Newborns with Mild Hypoxic-Ischemic Encephalopathy: A Retrospective Study. Ann Pediatr. 2021; 4(1): 1081. 
in $76 \%$ of un-cooled newborns vs $95 \%$ of cooled newborns. Most of the newborns have achieved full sucking power within 10 days (99\%). Cooled newborns had to stay longer in the NICU because of the added number of cooling where the length of stay was $11 \pm 4.7$ days vs $6.9 \pm 4.7$ days in un-cooled newborns. The MRI brain was done on 25 newborns, 12 MRIs were reported as abnormal (48\%) and consistent with hypoxic-ischemic changes, 5/97 in the uncooled cases and 7 in the cooled cases. Neurodevelopmental assessments at 12 months and 18 months of age were abnormal in 14/116 newborns (12\%).

Conclusion: The current assumptions about the benignity of mild form of HIE may not be accurate. More attention to this category of HIE, clear diagnostic criteria, longer clinical observation, and vigilant neurological assessment are all required.

\section{Introduction}

Hypoxic-Ischemic Encephalopathy (HIE) is one of the most unexpected devastating outcomes at birth. It is the result of brain deprivation of oxygen and glucose, secondary to either hypoxemia or ischemia. The clinical presentation depends on the severity, timing, and duration of the insult, with symptoms typically evolving over 72 hours. Brain injury begins with the initial hypoxic-ischemic event. Within 6 to 24 hours after the insult, there may be more deterioration, resulting in a secondary phase of energy failure. The severity of this delayed energy failure closely correlates with survival and neurodevelopmental sequelae [1].

HIE remains a significant cause of mortality and long-term disability in late preterm and term newborns. Therapeutic Hypothermia ( $\mathrm{TH})$, if initiated as soon as possible within the first $6 \mathrm{~h}$ of life, will significantly decrease mortality and severe longterm neurodevelopmental disabilities in newborns with moderate and severe HIE who are $\geq 36$ weeks' gestational age. It has minimal side effects, and the incidence of disability in survivors is not increased. TH can be achieved by either total body or selective head cooling. As cooling is now considered a standard of care, newborns $\geq 36$ weeks' gestational age who are depressed at birth should be assessed to determine whether they meet the criteria for $\mathrm{TH}$. There is currently no strong evidence that $\mathrm{TH}$ offers any benefit to mild HIE [2].

Mild to moderate cerebral hypothermia initiated before the secondary deterioration seems to offer neuroprotection in experimental animals and newborns. The neuroprotective effect decreases and might disappear if TH is delayed beyond 6 hours [3].

At birth, the only available distinction between mild, moderate, and severe HIE is based on the clinical ground, using either Sarnat and Sarnat's [4] or Thompson's stages of encephalopathy [5]. The three stages of HIE share similar intrapartum and postpartum events, but express different intensities, while electro-chemical changes are more evident in moderate and severe forms. Nevertheless, mild HIE can be presented with subtle or subjective clinical features that may mislead the treating physician and delay his decision to intervene. Indeed, "neonatal depression" is a general term occasionally used to describe the condition of any newborn showing a prolonged transition from intrauterine to extra-uterine life, in the immediate postnatal period but not yet fulfilling criteria of HIE. The variable degree of depression is inversely related to the Apgar score, with $1 \mathrm{~min}$. scores of 0 to 3 indicating the most severe forms, which may include perinatal asphyxia [6]. Heart rate variability was found to be a significant indicator of the severity of HIE, with Golden et al. reporting a statistically significant reduction in all heart rate variability measures in neonates with moderate and severe HIE compared with neonates with mild HIE [7]. Such vagueness may lead to a decision not to offer therapeutic hypothermia to those newborns who have suffered from mild HIE. Early neonatal hypothermia trials have been designed to enlist newborns with a high possibility of poor outcomes, while newborns with mild HIE have always been excluded. However, there has been no authorized recommendation to treat newborns who sustain mild HIE [8-10]. Recently, several publications have queried this. All except one of these [12], are retrospective observational trials. DuPont et al. demonstrated that $20 \%$ of newborns with perinatal acidosis, who were diagnosed with mild HIE, had abnormal short-term outcomes linked to their encephalopathies, such as seizures, abnormal MRI findings, abnormal neurologic examination at discharge, gastrostomy tube, feeding difficulties in the neonatal period and death. [11,12]. The PRIME study completed by Chalak et al. in 2018 suggested that neonates with mild HIE have worse developmental outcomes compared to their healthy term newborns, and that $16 \%$ of untreated newborns with mild HIE showed disabilities at 18-22 months [12]. The PRIME study was a prospective observational study that defined mild HIE as any infant $\geq 35$ weeks and $\leq 6 \mathrm{~h}$ of age with at least one of the following risks for encephalopathy: 1 . A history of the acute perinatal event, 2. a 10-min. Apgar score $\leq$ 6 , 3. a continued need for positive pressure ventilation for 10 min. or history of cardiopulmonary resuscitation, or 4 . a venous or arterial blood gas sample with $\mathrm{pH} \leq 7.00$ or base deficit $\leq 10$ $\mathrm{mmol} / \mathrm{L}$ [12]. In this study, we are exploring the outcome of mild HIE newborns delivered in our institute and documenting the abnormal MRI reports in those cases.

\section{Methods}

This is a retrospective descriptive study that examined all inborn newborns $\geq 35$ weeks gestational age born at a single, tertiary level Neonatal Intensive Care Unit (NICU) in women's hospital, Hamad Medical Corporation. We revised 265 newborns who were admitted to our NICU during the period from November 2014 till November 2020 under the diagnoses of HIE, birth depression, neonatal depression, birth asphyxia, neonatal asphyxia, asphyxia neonatorum, or even perinatal depression of the newborn. During the revision of these newborns, we excluded all newborns with moderate or severe HIE, unexplained neonatal seizure within the first 3 days of life, unexplained hypotonia or hyporeflexia, birth weight less than $2.5 \mathrm{kgs}$, gestation age less than 35 weeks gestation, and / or congenital anomalies.

The decision to start therapeutic hypothermia in cases of mild HIE was off-label and it was taken according to the clinical judgment of the treating team. The therapeutic hypothermia used in the cooled newborns was performed according to the same standards used for treating moderate and severe HIE newborns (i.e. same cooling machine, same target temperature for the same duration, and applying the same precautions).

International Classification of Disease codes were used to identify neonates with mild HIE and perinatal depression. To determine whether or not they should receive therapeutic hypothermia, we used similar inclusion criteria used in the PRIME study [12], which included the intrapartum fetal deficiency or poor maternal event before delivery, Apgar score, need for 
neonatal resuscitation at birth, cord blood gases, less than onehour blood gas measurement, clinical behavior of the newborn inside the NICU, and biochemical results indicating multi-organ failure. Sarnat and Sarnat's HIE classification [4] was used to distinguish mild from moderate or severe HIE cases. MRI reports were re-evaluated by a single qualified radiologist to ensure a unified MRI description.

Short - and long-term neurodevelopmental outcomes were documented during follow-up in neurodevelopmental clinics, routine patient follow-up clinics, and routine vaccination clinics.

The neurodevelopmental follow-up for these newborns would include five visits at 4, 8, 12, 18, and 24 months of age. Newborns would undergo three assessments tools: A General Motor Assessment (GMA) for the fidgety stage, Alberta Infant Motor Scale (AIMS), and Bayley infant screener III. We used the Ages and Stages Questionnaire 3 (ASQ-3) assessment for all newborns seen in the virtual clinic (during the Covid pandemic). In addition, we employed a parent questionnaire called the Social and Emotional Component of ASQ (ASQ-SE2) at 18 months and 24 months.

\section{Statistics}

Data analysis was performed with SPSS software, V.26.0.0.2 (SPSS Inc., Chicago, IL, USA). Continuous variables were presented as mean values Standard Deviation $( \pm S D)$. Categorical variables were presented as percentages. Sample distribution data are presented in percentages. The abnormal neurodevelopmental rate was calculated as a function of the variables total cooled and total un-cooled admissions, and presented as percentages.

\section{Ethical approval}

The data for this study were retrieved from PEARL Maternal \& Newborn Registry and WWRC medical records. IRB / Ethical approval was granted with a waiver of consent.

\section{Results}

Out of the 265 newborns admitted with a history suggestive of HIE or neurological deficits, only 116 newborns matched the diagnosis of mild HIE according to the above-mentioned exclusions. A total of 19 newborns out of the 116 mild HIE cases received therapeutic hypothermia. Antepartum and / or intrapartum complications were recorded in 48 mothers, including infants of insulin-dependent diabetic mothers (12), pre-eclampsia (3), cord prolapse (2), shoulder dystocia (2), antepartum hemorrhage (8), chorioamnionitis (6), poor CTG tracing (13), and ruptured uterus (2).

Mean gestation was $38 \pm 2$ weeks, mean birth weight was $3.0 \pm 0.5 \mathrm{~kg}$, Cesarean section was $57 \%$ in the un-cooled group vs $75 \%$ in the cooled group. Mean Apgar score at 10 th minute was $7.9 \pm 1.8$ vs $5.3 \pm 2.2$ in the un-cooled vs cooled group, the $\mathrm{p}$-value is 0.002 . Arterial cord $\mathrm{pH}$ was $7.15 \pm 0.3$ vs $6.92 \pm 0.26$. The base deficit in the first-hour blood gas was $-7.83 \pm 5$ vs $-12 \pm 5.6(p=0.005)$. The total number of cooled newborns was 19 (16\%). Respiratory support was required in $76 \%$ of un-cooled newborns vs $95 \%$ of cooled newborns. Most of the newborns achieved full sucking power within 10 days (99\%). Cooled newborns had to remain longer in the NICU because of the time required for the cooling, where the length of stay was $11 \pm 4.7$ days vs $6.9 \pm 4.7$ days in un-cooled newborns. The MRI brain scan was done on 25 newborns, with 12 reported as abnormal (48\%) and consistent with hypoxic-ischemic changes, 5/97 in the uncooled cases and 7 in the cooled cases. Neurodevelopmental assessments at 12 months and 18 months of age were abnormal in 14/116 newborns (12\%).

\section{Discussion}

Therapeutic hypothermia is standard care for those newborns with moderate to severe HIE [9-13]. However, there is no agreement regarding mild HIE $[12,14]$. This study was conducted after observing that some newborns who have been diagnosed with mild HIE or diagnosed as trivial neonatal depression at birth become neurologically affected and reported to have abnormal MRI with ischemic changes and/or abnormal neurologic examination at the time of discharge. In this study, we applied several criteria to filter those newborns who can be diagnosed with mild HIE. These included trivial adverse intrapartum events combined with need for short resuscitation of less than 10 min. with rapid improvement, border-line Apgar score, cord gas, and first-hour blood gas. In addition, the clinical behavior inside the NICU was an important parameter of the extent to which the baby was affected, based on Sarnat and Sarnat's staging, and hemodynamic and respiratory stability. The decision of whether to offer hypothermia treatment was made by the attending physician in such a scenario. A total of $16 \%$ of cases in the study received hypothermia therapy. At 18 months of age, abnormal neurodevelopmental examinations were recorded in $9 \%(9 / 97)$ of the un-cooled versus $26 \%(5 / 19)$ in the cooled cases. Although MRI was performed on only 25 cases before discharge, the brain MRI results reported abnormalities suggestive of ischemic changes in $48 \%$ of cases $(12 / 25)$, most of them in the cooled group (Figure 1). The MRI reports were delivered by different radiologists, and needed to be re-evaluated by a single radiologist (Table 3 ). Mild HIE is an important diagnosis, accounting for $50 \%$ of all cases of $\mathrm{HIE}$, as most newborns diagnosed with hypoxic brain injuries do not meet standard clinical criteria for moderate or severe encephalopathy [13]. Since therapeutic hypothermia was introduced as standard therapy, mild HIE was excluded [14]. However, quite a few clinicians are offering therapeutic hypothermia to those newborns $[15,16]$.

Finder et al., in a large multi-center retrospective cohort study including 471 newborns, determine that children with mild HIE at birth have significantly lower cognitive composite scores than healthy controls [17]. In addition, some evidence suggests that those newborns are at increased risk of adverse outcomes, including abnormal discharge examination, abnormal aEEG, hypoxic-ischemic changes in the MRI, and long-term NDI if left untreated [15]. In a systemic review that included 20 studies, Conway et al. identified an abnormal ND outcome in $86 / 341$ (25\%) of affected newborns. There was insufficient evidence to examine the effect of TH on the outcome [18]. Similar findings were addressed in a prospective multi-center cohort of 54 newborns with mild HIE who were not treated with hypothermia. Newborns were identified on the basis of abnormal aEEG within the first 9 hours of life, where 28 newborns had abnormal brain MRI and clinical evidence of NDI [12].

There is growing evidence that while mild HIE may initially appear to be mild, due to difficulties in measuring cognitive ability at a very young age, problems may appear as children mature to school age and beyond. A significant proportion (approximately $25 \%$ ) of newborns with mild HIE have mild to moderate disability at 2 years of age, and by 5 years, 35\% will show difficulties in one or more areas [19-21]. 
Table 1

\begin{tabular}{|c|c|c|c|c|c|c|c|}
\hline & \multicolumn{6}{|c|}{ Cooling Therapy } & \multirow{3}{*}{$P$ value } \\
\hline & \multicolumn{2}{|c|}{$\begin{array}{c}\text { No }(n=97) \\
n(\%) \text { or mean } \pm S D^{*}\end{array}$} & \multicolumn{2}{|c|}{$\begin{array}{c}\text { Yes }(n=19) \\
n(\%) \text { or mean } \pm S D^{*}\end{array}$} & \multicolumn{2}{|c|}{$\begin{array}{c}\text { Total }(n=116) \\
n(\%) \text { or mean } \pm S D^{*}\end{array}$} & \\
\hline & $\mathrm{N}$ & $\%$ & $\mathrm{~N}$ & $\%$ & $\mathrm{~N}$ & $\%$ & \\
\hline Maternal age & 28 & \pm 12 & 29 & \pm 12 & 28 & \pm 12 & 0.9 \\
\hline \multicolumn{7}{|l|}{ Baby gender } & \multirow[t]{3}{*}{0.508} \\
\hline Male & 51 & 53.1 & 9 & 45 & 60 & 51.7 & \\
\hline Female & 45 & 46.9 & 11 & 55 & 56 & 48.3 & \\
\hline Gestational age (GA)* & 38.39 & 2.08 & 37.95 & 1.67 & 38.31 & 2.01 & 0.382 \\
\hline \multicolumn{7}{|l|}{ Mode of delivery } & \multirow[t]{3}{*}{0.141} \\
\hline Vaginal delivery & 41 & 42.7 & 5 & 25.0 & 46 & 39.7 & \\
\hline Cesarean-section & 55 & 57.3 & 15 & 75.0 & 70 & 60.3 & \\
\hline Birth Weight $(\mathrm{g})^{*}$ & 3100.14 & \pm 603.6 & 3103 & 504.85 & 3017.87 & \pm 587.03 & 0.478 \\
\hline \multicolumn{7}{|l|}{ Multiple Pregnancy } & 0.080 \\
\hline Single & 93 & 97.9 & 18 & 90 & 111 & 96.5 & \\
\hline Multiple & 2 & 2.1 & 2 & 10 & 4 & 3.5 & \\
\hline Apgar score 1st & 4.77 & \pm 4.7 & 2.22 & \pm 1.35 & 4.37 & \pm 4.44 & 0.025 \\
\hline Apgar score 5th & 7.3 & \pm 1.86 & 5.5 & \pm 2.18 & 6.4 & \pm 2.09 & 0.000 \\
\hline Apgar score 10th & 7.7 & \pm 1.82 & 5.3 & \pm 2.26 & 7.09 & \pm 2.27 & 0.002 \\
\hline Cord pH - arterial * & 7.15 & \pm 0.32 & 6.92 & \pm 0.26 & 7.1 & \pm 0.33 & 0.007 \\
\hline First gas $\mathrm{pH}^{*}$ & 7.29 & \pm 0.13 & 7.16 & \pm 0.13 & 7.26 & \pm 0.14 & 0.000 \\
\hline First gas lactate* & 22 & \pm 10.95 & 6.83 & \pm 9.87 & 18.75 & \pm 5.81 & 0.590 \\
\hline Cord gas base deficit & -9.03 & \pm 5.03 & -13.57 & \pm 7.7 & -9.77 & \pm 5.75 & 0.005 \\
\hline First gas base deficit & -7.82 & \pm 5.66 & -12.32 & \pm 5.63 & -8.7 & \pm 5.9 & 0.007 \\
\hline \multicolumn{8}{|l|}{ Delivery room Resuscitation } \\
\hline IPPV $\geq 5$ minutes & 24 & 24.7 & 12 & 63 & 36 & 31 & \\
\hline Intubation & 24 & 24.7 & 12 & 63 & 36 & 31 & \\
\hline Cardiac Massage and drugs resuscitation & 0 & 0 & 0 & 0 & 0 & 0 & NA \\
\hline \multicolumn{7}{|l|}{ Types of respiratory support in the NICU } & 0.087 \\
\hline No support & 24 & 24 & 0 & 0 & 24 & 20 & \\
\hline CPAP & 19 & $20 \%$ & 5 & 26 & 24 & 26.4 & \\
\hline Nasal cannula & 29 & 30 & 1 & 5 & 34 & 29 & \\
\hline NIPPV & 1 & 0.9 & 1 & 5 & 2 & 1.7 & \\
\hline Mechanical ventilation & 24 & 24.7 & 12 & 63 & 33 & 36.3 & \\
\hline Days for full sucking* & 3.89 & \pm 4 & 6.25 & 4.17 & \pm 4.29 & 4.11 & 0.019 \\
\hline Full oral sucking on discharge & & & & & & & 0.647 \\
\hline Yes & 95 & 99 & 20 & 100 & 115 & 99.1 & \\
\hline No & 1 & 1 & 0 & 0 & 1 & 0.9 & \\
\hline Length of stay (LOS)* & 6.89 & \pm 7.65 & 11.15 & \pm 4.66 & 7.62 & \pm 7.39 & \\
\hline Brain MRI Result ( $n=25 / 116)$ & & & & & & & 0.31 \\
\hline Normal & $3 / 25$ & $12 \%$ & $12 / 25$ & 48 & $13 / 25$ & 40 & \\
\hline Abnormal & $5 / 25$ & $20 \%$ & $7 / 25$ & 28 & $12 / 25$ & 48 & \\
\hline Abnormal ${ }^{\&}$ NDA at $12 \& 18$ months of age & 9/96 & $9 \%$ & $5 / 19$ & 26 & $14 / 115^{* *}$ & 12 & 0.508 \\
\hline
\end{tabular}

${ }^{*}$ Values expressed as mean \pm Standard Deviation (SD). P value values denote statistical significance at the $p<0.05$ level. ${ }^{* *}$ one patient left the country at one month of age \& NDA: Neurodevelopmental assessment. 
Table 2: MRI findings in the $12 / 25$ cases with mild HIE.

1 Multifocal subtle abnormal signal intensity of the parieto-occipital and peri-trigonal white matter suggestive of post hypoxic-ischemic changes clinical correlation is indicated.

2 Voluminous basal ganglia with bilateral symmetric Globus pallidus T1 hyperintensity suggestive of post hypoxic ischemic insult.

3 Multifocal parenchymal hemorrhagic changes with ventricular extension. Hypoxic ischemic pattern of bilateral PLIC involvement.

4 Minimal frontal and parietal periventricular white matter edema with bright $\mathrm{T} 2$ and Flair suppression, noted most likely corresponding to hypoxic-ischemic insult.

Mild diffusion restriction in the splenium of the corpus callosum and adjacent periventricular region is likely transient splenial lesion due to post ictal changes of the known seizures.

Right high frontal few micro hemorrhages and high T1 signal intensity of the posteromedial lentiform nuclei suggestive of subacute post hypoxic ischemic insult.

${ }_{7}^{5}$ Multiple but small hemorrhagic lesions in the right posterior temporal and posterior parietal intraparenchymal region with adjacent subarachnoid hemorrhage which might be due to trauma clinical correlation is required.

8 Mild periventricular brain insult likely related to hypoxic ischemic brain injury. Tiny cerebellar foci of microhemorrhages are also noted.

9 Bilateral Globus pallida and ventrolateral thalami showed relative increased T1 signal in comparison to myelinated posterior limb of internal capsule, denoting mild element of hypoxic changes, also noted mild frontoparietal periventricular white matter edema with increased T2 signal and suppression on FLAIR.

10 Right frontal white matter recent ischemic focus and slight increase T1 of the basal ganglia suggestive of hypoxic ischemic encephalopathy.

11

Subtle abnormal signal intensity of the parieto-occipital and peri-trigonal white matter with prominent lactate peak seen at the peri-trigonal white matter could represent sequel of hypoxic changes

12 Bilateral cerebral predominantly right-sided frontal and bilateral occipital as well as right basal ganglia, thalamo-capsular and cerebral peduncles ischemic changes

$\S M R I$ report did not identify ischemic changes.

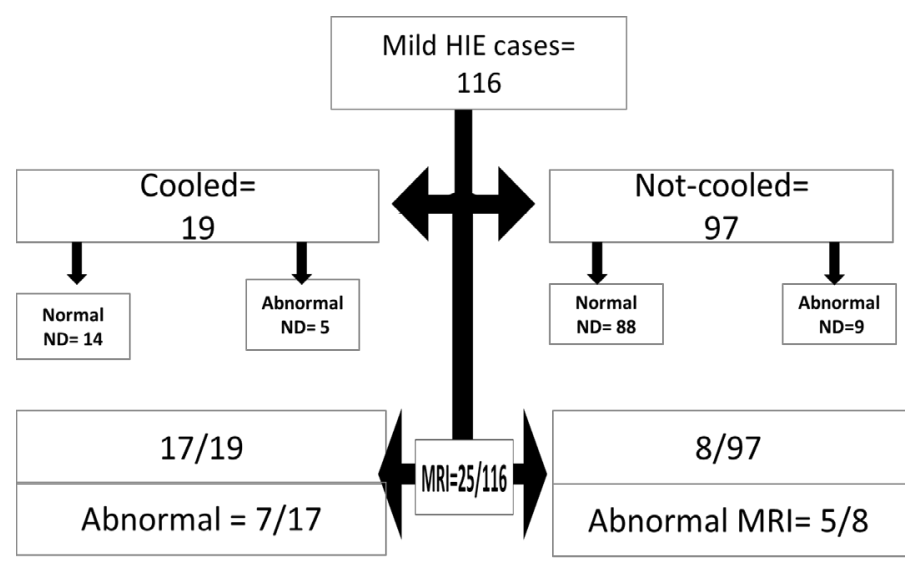

Figure 1: Newborns distribution.

\section{Limitations}

In this retrospective study, the neurodevelopmental followup tools applied in the clinic are not consistent, and not all tests were applied in all cases, with the Bayley developmental assessment being applied at an especially limited rate. Performing brain MRI for only 25 cases was highly selective based on the clinical presentations. Amplitude EEG was measured in only a few cases, which represented the omission of important neuroelectrical diagnostic data. Lack of aEEG measurements can be attributed to the perception of benignity of the condition.

\section{Conclusion}

In this retrospective study, 12 brain MRI findings suggestive of brain insults were described, representing $10 \%$ of all cases in the study and $48 \%$ of cases who received an MRI brain investigation. In addition, the rate of NDI at 12 and 18 months was $12 \%$ of all cases. These figures should be of concern and must raise alarms regarding the current assumptions about the benignity of this form of HIE. More attention to this category of HIE, clear diagnostic criteria, longer clinical observation, and vigilant neurological assessment are all required. Moreover, prospective randomized trials are pressing to answer two main questions: Are the longer-term outcomes of mild HIE genuinely mild; and is therapeutic hypothermia justifiable?.

\section{References}

1. Ferriero DM. Neonatal brain injury. N Engl J Med. 2004; 351: 1985-95.

2. Shalak L, Perlman JM. Hypoxic-ischemic brain injury in the term infant - current concepts. Early Hum Dev 2004; 80: 125-41.

3. Jacobs S, Hunt R, Tarnow-Mordi W, Inder T, Davis P. Cooling for newborns with hypoxic ischemic encephalopathy. Cochrane Database Syst Rev. 2007; 4: CD003311. Update in Cochrane Database Syst Rev. 2013; 1: CD003311.

4. Sarnat HB and Sarnat MS. Neonatal encephalopathy following fetal distress: A clinical and electro-encephalographic study. Arch neurol. 1976; 33: 696-705.

5. Thompson CM, Puterman AS, Linley LL, Hann FM, van der Elst $\mathrm{CW}$, et al. The value of a scoring system for hypoxic-ischemic encephalopathy in predicting neurodevelopmenal outcome. Acta Pediatr. 1997; 86: 757-761.

6. Paolo Biban and Davide Silvagni. Early Detection of Neonatal Depression and Asphyxia. Neonatology. Neonatology. 2020.

7. Goulding RM, Stevenson NJ, Murray DM, Livingstone V, Filan $\mathrm{PM}$, et al. Heart rate variability in hypoxic ischemic encephalopathy: correlation with EEG grade and 2-y neurodevelopmental outcome. Pediatr Res. 2015; 77: 681-687. 
8. Azzopardi DV, Strohm B, Edwards AD, Dyet L, Halliday HL, et al. Moderate hypothermia to treat perinatal asphyxial encephalopathy. N Engl J Med. 2009; 361: 1349-1358.

9. Sankaran S, Laptook AR, Ehrenkranz RA, Tyson JE, McDonald SA, et al.; National Institute of Child Health and Human Development Neonatal Research Network. Whole-body hypothermia for neonates with hypoxic-ischemic encephalopathy. N Engl J Med. 2005; 353: 1574-1584.

10. Nair J, Kumar VH. Current and Emerging Therapies in the Management of Hypoxic Ischemic Encephalopathy in Neonates. Children (Basel). 2018; 5: E99.

11. DuPont TL, Chalak LF, Morriss MC, Burchfield PJ, Christie L, et al. Short-term outcomes of newborns with perinatal acidemia who are not eligible for systemic hypothermia therapy. J Pediatr. 2013; 162: 35-41.

12. Chalak LF, Nguyen KA, Prempunpong C, Heyne R, Thayyil S, et al. Prospective research in newborns with mild encephalopathy identified in the first six hours of life: neurodevelopmental outcomes at 18-22 months. Pediatr Res. 2018; 84: 861-868.

13. Murray DM, O'Connor CM, Ryan CA, Korotchikova I, Boylan GB. Early EEG Grade and Outcome at 5 Years After Mild Neonatal Hypoxic Ischemic Encephalopathy. Pediatrics. 2016; 138: e20160659.

14. Martinello K, Hart AR, Yap S, Mitra S, Robertson NJ. Management and investigation of neonatal encephalopathy. 2017 update. Arch Dis Child Fetal Neonatal Ed. 2017; 102: F346-F358.
15. Lodygensky GA, Battin MR, Gunn AJ. Mild Neonatal Encephalopathy-How, When, and How Much to Treat?. JAMA Pediatric. 2018; 172: 3-4.

16. Saw CL, Rakshasbhuvankar A, Rao S, Bulsara M, Patole S. Current Practice of Therapeutic Hypothermia for Mild Hypoxic Ischemic Encephalopathy. J Child Neurol. 2019; 34: 402-409.

17. Finder M, Boylan GB, Twomey D, Ahearne C, Murray DM, et al. Two-Year Neurodevelopmental Outcomes After Mild Hypoxic Ischemic Encephalopathy in the Era of Therapeutic Hypothermia. JAMA Pediatr. 2020; 174: 48-55.

18. Conway JM, Walsh BH, Boylan GB, Murray DM. Mild hypoxic ischaemic encephalopathy and long term neurodevelopmental outcome - A systematic review. Early Hum Dev. 2018; 120: 8087.

19. Reiss J, Sinha M, Gold J, Bykowski J, Lawrence SM. Outcomes of Newborns with Mild Hypoxic Ischemic Encephalopathy Who Did Not Receive Therapeutic Hypothermia. Biomed Hub. 2019; 4: 1-9.

20. Murray DM, O'Connor CM, Anthony Ryan C, Korotchikova I, Boylan GB. Early EEG grade and outcome at 5 years after mild neonatal hypoxic ischemic encephalopathy. Pediatrics. 2016; 138.

21. Van Kooij BJ, van Handel M, Nievelstein RA, Groenendaal F, Jongmans MJ, et al. Serial MRI and neurodevelopmental outcome in 9- to 10-year-old children with neonatal encephalopathy. J Pediatr. 2010; 157: 221-227. 